Review of atomic data needs for active charge-exchange spectroscopy on ITER

O. Marchuk, G. Bertschinger, W. Biel, E. Delabie, M. G. von Hellermann, R. Jaspers, and D. Reiter

Citation: Review of Scientific Instruments 79, 10F532 (2008);

View online: https://doi.org/10.1063/1.2965019

View Table of Contents: http://aip.scitation.org/toc/rsi/79/10

Published by the American Institute of Physics

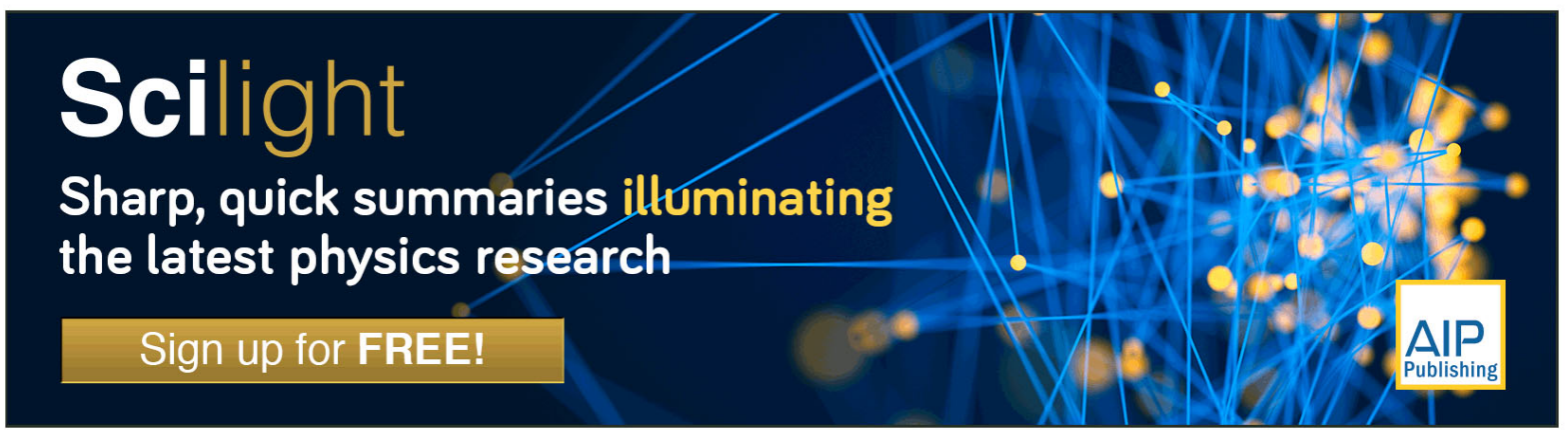




\title{
Review of atomic data needs for active charge-exchange spectroscopy on ITER ${ }^{\mathrm{a})}$
}

\author{
O. Marchuk ${ }^{1, b)}$ G. Bertschinger, ${ }^{1}$ W. Biel, ${ }^{1}$ E. Delabie, ${ }^{2}$ M. G. von Hellermann, ${ }^{2}$ \\ R. Jaspers, ${ }^{2}$ and D. Reiter ${ }^{1}$ \\ ${ }^{1}$ Institut fuer Energieforschung-Plasmaphysik, Forschungszentrum Juelich, 52425 Jülich, Germany and \\ Association EURATOM-FZJ, 52425 Jülich, Germany \\ ${ }^{2}$ FOM-Rijnhuizen, Association EURATOM-FOM, P.O. Box 1207, 3430 BE Nieuwegein, The Netherlands
}

(Presented 15 May 2008; received 9 May 2008; accepted 7 July 2008; published online 31 October 2008)

\begin{abstract}
The quantitative exploitation of active beam spectra is largely based on an advanced atomic modeling. Under the ITER operating conditions the penetration depth of a diagnostic beam into the plasma core crucially affects the intensities of spectral lines and hence the uncertainties of derived plasma parameters. A critical review of atomic data and an assessment of its error margins are, therefore, urgently needed. The aim of the present work is to verify the existing beam-stopping and beam-emission data for hydrogen beam in fusion plasmas. The agreement between the ADAS database and the present calculations is found to be within 5\% for the beam-stopping data in a $\mathrm{H}$-plasma. The calculation of beam attenuation in the presence of He-ash (4\%) and Be ions (2\%) demonstrates the agreement between the present data and the ADAS database within $10 \%$. Finally, the maximum deviation of $15 \%$ is found only for beam-emission data at the electron density of $1 \times 10^{12}-2 \times 10^{12} \mathrm{~cm}^{-3}$, which is significantly below the ITER density of $10^{14} \mathrm{~cm}^{-3}$. (C) 2008 American Institute of Physics. [DOI: 10.1063/1.2965019]
\end{abstract}

\section{INTRODUCTION}

The present design of ITER incorporates only a few diagnostic systems capable of providing information on plasma core characteristics such as ion temperature and plasma rotation or density of impurities. So, for example, the He-ash density measurement is required to $10 \%$ accuracy at the time resolution of $100 \mathrm{~ms}$ and the measurements of ion temperature are required to $5 \%$ accuracy within $10 \mathrm{~ms} .^{1}$ The only diagnostic meeting such challenging needs to the quality of measured plasma parameters is the active charge-exchange spectroscopy. Such quantitative evaluation of chargeexchange spectra can be performed, however, only on a verified set of atomic constants used at the spectra modeling.

The atomic data incorporated in the ADAS database ${ }^{2}$ has been extensively used for charge-exchange and XUV diagnostics on fusion devices such as JET, ${ }^{3}$ ASDEX, ${ }^{4}$ TEXTOR, ${ }^{5}$ or TORE SUPRA. ${ }^{6}$ The assessment of the quality of these data can be done either on the base of experimental results or by means of independent calculations including multistep processes. ${ }^{7}$ So, recently, for example, a seeming inconsistency was pointed out between the ADAS data and calculations ${ }^{8}$ for the population density of the excited $n=3$ states. The reason for this discrepancy turned out to be a different set of fundamental data used in calculation. On the other hand, the experiment on Alcator-C Mod pointed out the importance of the charge-exchange capture from the excited $n=3$ states for the boron $\mathrm{B}^{5+}$ ions. ${ }^{9}$

\footnotetext{
a) Contributed paper, published as part of the Proceedings of the 17th Topical Conference on High-Temperature Plasma Diagnostics, Albuquerque, New Mexico, May 2008.

b)Electronic mail: o.marchuk@fz-juelich.de.
}

The aim of this paper is to verify the quality of beamstopping cross section (BSCS) and beam-emission cross sections (BECS) for a diagnostic hydrogen beam at ITER conditions. In contrast to all the present devices, less than $2 \%$ of the beam penetrates into the plasma core, so that the verification of the atomic data of the beam attenuation becomes the urgent task of the port plug design.

\section{OVERVIEW OF DIAGNOSTIC NEEDS}

The charge-exchange port plug designed for ITER is demonstrated in Fig. 1. In the present scheme the hydrogen beam with the energy of $100 \mathrm{keV} /$ amu penetrates almost radially into the plasma core with the electron density of about $10^{14} \mathrm{~cm}^{-3}$ and the electron and ion temperatures of $20 \mathrm{keV}$. The distance from the beam entrance point to the plasma core is about $2 \mathrm{~m}$. The input set of atomic data used by the diagnostic includes the BSCS responsible for the neutral beam attenuation in the plasma. The density of neutral beam at the distance $x$ from the entrance can be written as follows:

$$
N_{b}(x)=N_{b 0} \exp \left(-\int_{0}^{x} \sigma_{\mathrm{BS}}(z) N_{e}(z) d z\right),
$$

where $N_{b 0}$ is the neutral beam density at the entrance, $N_{e}$ is the electron density and $\sigma_{\mathrm{BS}} \equiv \sigma\left(E_{b}, N_{e}, T_{e}, T_{i},\left\{n_{j}\right\}\right)$ is the BSCS. The latter depends on the beam energy $E_{b}$, electron density $N_{e}$ and temperature $T_{e}$, and density of ions $\left\{n_{j}\right\}$ with the temperature $T_{i}$. The BSCS is determined by excitation/ ionization of hydrogen atoms by electrons and ions and by charge-exchange capture by ions. 


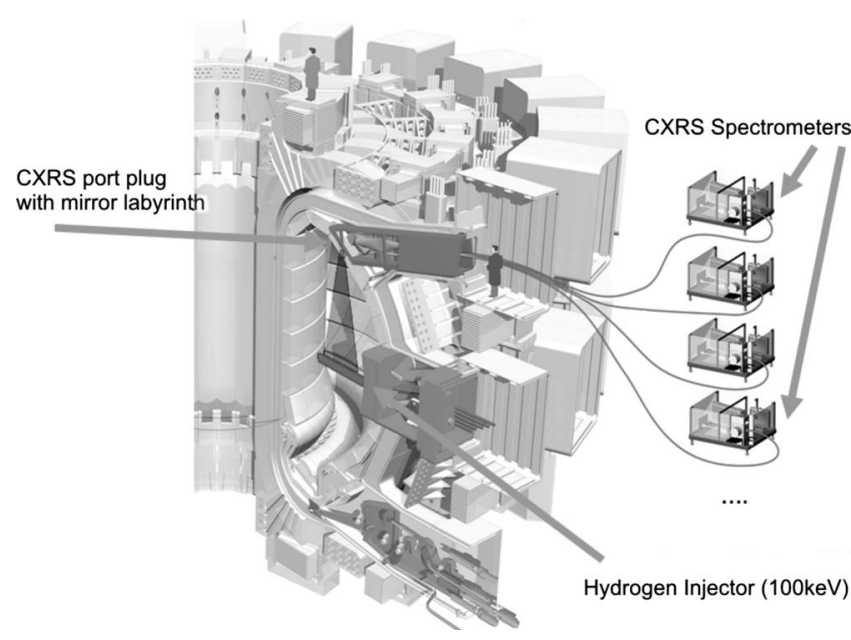

FIG. 1. Overview of the CXRS port plug for ITER (Ref. 10) (based on an ITER drawing).

The second set of data describes the intensity of spectral lines. The intensity of a spectral line $I_{L}$ due to chargeexchange capture into an excited state of a specific impurity with the density $n_{Z}$ is determined as follows:

$$
I_{L}(x)=\int_{\text {l.o.s. }} N_{b} n_{Z}\left\langle\sigma_{\mathrm{CX}}\left(n, n^{\prime}\right) \cdot u\right\rangle d l,
$$

where the integral is taken along the instrumental line of sight, $\sigma_{\mathrm{CX}}\left(n, n^{\prime}\right)$ is the effective cross section of a specific transition between the states $n$ and $n^{\prime}$, and $u$ is the relative velocity between the neutral beam particle and impurity ion. For example, the He II $(n=4, n=3)$, Be IV $(n=6, n=5)$, Ne X $(n=11, n=10)$, and Ar XVIII $(n=16, n=15)$ transitions are considered as candidates for the spectrometer design for the ITER. The further technical specification of the design layout can be found, for example in Refs. 10 and 11.

The uncertainties in the cross sections in Eqs. (1) and (2) have different effects. The errors in Eq. (1) propagate along the beam path and consequently the errors of $10 \%$ will considerably change the density of neutral particles at the center. Indeed, the e-folding length of the beam at the plasma core is $L_{e}=1 / N_{e} / \sigma_{\mathrm{BS}} \approx 0.3-0.5 \mathrm{~m}$ and the errors of $10 \%$ in the $\sigma_{\mathrm{BS}}$ will change the neutral density in the core by a factor of 2 . In contrast, the signal of a spectral line changes linearly with the uncertainties in the cross sections in Eq. (2). In this paper the comparison of the first set of the cross sections for the hydrogen beam will be performed. The validation of the change-exchange cross sections of impurities is the aim of a future investigation.

\section{CALCULATION OF BEAM-STOPPING CROSS SECTIONS}

The BSCS, were calculated using the collisionalradiative model for excited states of a hydrogen beam as follows:

$$
\frac{\partial N_{p}}{\partial t}+V_{b} \frac{\partial N_{p}}{\partial x}=\sum_{p \neq l} N_{l} W_{l p}-N_{p}\left(\sum_{p \neq l} W_{p l}+N_{e} S_{p}^{i}\right),
$$

where

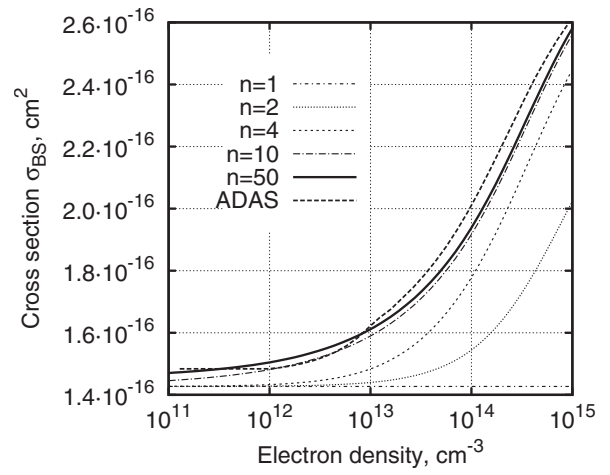

FIG. 2. Steady-state calculations of BSCS for hydrogen plasma. The results are demonstrated for the different number of excited states included in the model. The ADAS data are shown as the dashed line. The beam energy was taken at $100 \mathrm{keV} / \mathrm{amu}$ and electron and ion temperatures were taken at $20 \mathrm{keV}$.

$$
\begin{aligned}
& W_{l p}=N_{e}\left(k_{l p}^{e e}+\sum_{j} c_{j} k_{l p}^{i e(j)}\right)+A_{l p}, \\
& S_{p}^{i}=k_{p}^{e i}+\sum_{j} c_{j}\left(k_{p}^{i i(j)}+k_{p}^{i c x(j)}\right) .
\end{aligned}
$$

Here, $N_{p}$ is the density of the excited state of hydrogen $\left(\Sigma_{p} N_{p}=N_{b}\right), V_{b}$ is the beam velocity, and $c_{j}=n_{j} / N_{e}$ is the abundance of the impurity ion $j$ in plasma. The term $W_{l p}$ describes the contribution from any state $l$ to $p$ and $S_{p}^{i}$ is the term responsible for ionization and charge exchange from the state $p$. The $A_{l p}$ is the radiative rate of the transition from the state $l$ to $p$, and $k_{p l}^{e e, i e}$ are the excitation $(p<l)$ or deexcitation $(l<p)$ rates due to electron $(e e)$ or ion collisions (ie). The rates $k_{p}^{e i, i i}$ are the electron and ion ionization rates, respectively. The ionization of the state $p$ due to chargeexchange process was taken into account with the rate coefficients $k_{p}^{i c x}$. The cross sections for all collisional processes were taken from Ref. 12 and averaged over the relative velocities of the beam and plasma particles. The radiative rates for $n \leqslant 6$ were taken from the NIST database ${ }^{13}$ and hydrogenic approximation was assumed for transitions higher than $n \leqslant 50$. $^{14}$

Using Eqs. (1), (3), and (5) BSCS can be defined using the following expression: $\sigma_{\mathrm{BS}}=\Sigma_{p} N_{p} S_{p} / N_{b} / V_{b}$. The BECS

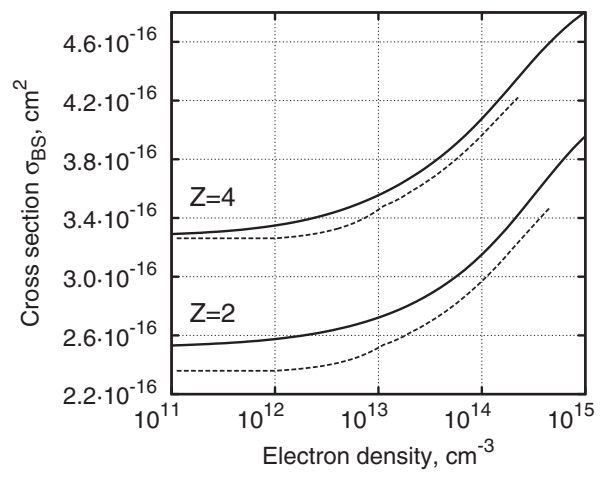

FIG. 3. The BSCS for helium and beryllium plasmas. The number of excited states included in the model is $n=50$. The beam energy was taken at $100 \mathrm{keV} / \mathrm{amu}$, and electron and ion temperatures were taken at $20 \mathrm{keV}$. The solid lines are based on the present data and the dashed lines are based on the ADAS data. 


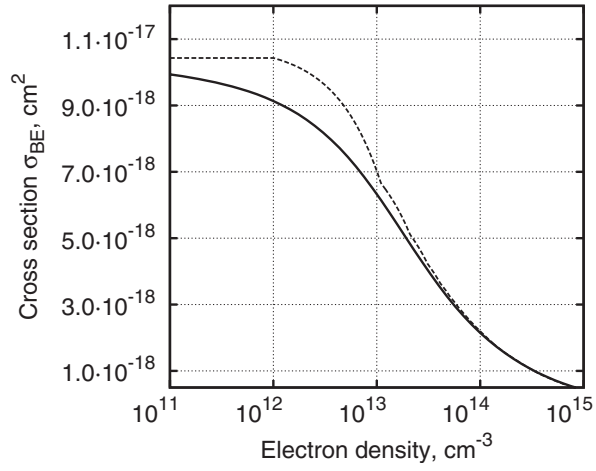

FIG. 4. The beam-emission data for hydrogen plasma. The beam energy was taken at $100 \mathrm{keV} / \mathrm{amu}$, and electron and ion temperatures were taken at $20 \mathrm{keV}$. The solid line is the present results and the dashed line is the ADAS data.

for the $n=3 \rightarrow n=2$ transition, also measured in tokamak plasmas, ${ }^{3}$ is determined as $\sigma_{\mathrm{BE}}=N_{3} A_{32} / N_{b} / V_{b}$. The BSCS for a hydrogen beam penetrating hydrogen plasma is shown in Fig. 2. The calculations were performed up to $n=50$; however, the results converge within 5\% taking only the first $n$ $=10$ states. The agreement with the ADAS data for hydrogen is also obtained within 5\%. The results of comparison for the hydrogen beam penetrating in the $\mathrm{He}$ - and Be-plasmas are presented in Fig. 3. Here, a deviation of $10 \%$ in the cross sections is observed only for the low-density case. The BSCS in a plasma with different impurity concentrations can be calculated using the following relation: $\sigma_{\mathrm{BS}}=\Sigma_{j} c_{j} \sigma_{\mathrm{BS}}^{j}$, where $\sigma_{\mathrm{BS}}^{j}$ is the cross section in pure $\mathrm{H}-, \mathrm{He}-$, or Be-plasmas.

The results for the beam-emission data in hydrogen plasma are shown in Fig. 4. We note the difference of about $15 \%$ observed for the density of $(1-2) \times 10^{13} \mathrm{~cm}^{-3}$. Both sets of data are, however, in good agreement with the higher densities relevant to ITER.

\section{CONCLUSIONS}

In this paper the beam attenuation atomic data used for the design of active charge-exchange spectroscopy at ITER (Ref. 11) are presented. The difference in the BSCS between the present calculation and the ADAS data was found to be within $7 \%$ for plasma parameters expected for the ITER core. This agreement is quite satisfactory. The discrepancy of about $15 \%$ for the BECS is found only at low electron densities and hence an additional experimental verification of the beam-emission data is necessary.

${ }^{1}$ B. Schunke, CXRS, and BES on ITER, An update of performance studies, ITER organization, ITER_D_2FF69X, https://user.iter.org (2008).

${ }^{2}$ H. P. Summers, The ADAS User Manual (2004), version 2.6, http:// adas.phys.strath.ac.uk.

${ }^{3}$ H. Anderson, M. G. von Hellermann, R. Hoekstra, L. D. Horton, A. C. Howman, R. W. T. Konig, R. Martin, R. E. Olson, and H. P. Summers, Plasma Phys. Controlled Fusion 42, 781 (2000).

${ }^{4}$ E. Wolfrum, J. Schweinzer, M. Reich, L. D. Horton, and C. F. Maggi, Rev. Sci. Instrum. 77, 033507 (2006).

${ }^{5}$ U. Gerstel, L. Horton, H. P. Summers, M. G. von Hellermann, and B. Wolle, Plasma Phys. Controlled Fusion 39, 737 (1997).

${ }^{6}$ W. R. Hess, J. L. Farjon, R. Guirlet, and M. Druetta, Rev. Sci. Instrum. 73, 1775 (2002).

${ }^{7}$ R. Janev, C. Boley, and D. Post, Nucl. Fusion 29, 2125 (1989).

${ }^{8}$ I. H. Hutchinson, Plasma Phys. Controlled Fusion 44, 71 (2002).

${ }^{9}$ I. O. Bespamyanov, W. L. Rowan, R. S. Granetz, and D. F. Beals, Rev. Sci. Instrum. 77, 10F123 (2006).

${ }^{10} \mathrm{~W}$. Biel et al., Proceedings of the IAEA International Workshop, Jaipur, India, 20-22 February 2008 (unpublished).

${ }^{11}$ M. G. von Hellermann, R. J. E. Jaspers, E. Delabie, W. Biel, O. Marchuk, C. Giroud, N. C. Hawkes, and K.-D. Zastrow, Proceedings of the IAEA International Workshop, Jaipur, India, 20-22 February 2008 (unpublished).

${ }^{12}$ R. K. Janev and J. J. Smith, Suppl. J. Nucl. Fusion 4, 1 (1993).

${ }^{13}$ Yu. Ralchenko, A. Kramida, J. Reader, and NIST ASD Team, NIST Atomic Spectra Database, version 3.1.5, http://physics.nist.gov/asd3.

${ }^{14}$ R. D. Cowan, The Theory of Atomic Structure and Spectra (University of California Press, Berkeley, California, 1981), p. 440. 